\title{
Speed Control Method of Electric Vehicle for Improving Passenger Ride Quality
}

\author{
Hidetake Fuse ${ }^{1}$, Tohru Kawabe², Masayuki Kawamoto ${ }^{3}$ \\ ${ }^{1}$ Chiyoda Corporation, Yokohama, Japan \\ ${ }^{2}$ Faculty of Engineering, Information and Systems, University of Tsukuba, Tsukuba, Japan \\ ${ }^{3}$ International Innovation Interface Organization, University of Tsukuba, Tsukuba, Japan \\ Email: fuse.hidetake@chiyodacorp.com,kawabe@cs.tsukuba.ac.jp, kawamoto.masayuki.gn@un.tsukuba.ac.jp
}

How to cite this paper: Fuse, H., Kawabe, T. and Kawamoto, M. (2017) Speed Control Method of Electric Vehicle for Improving Passenger Ride Quality. Intelligent Control and Automation, 8, 29-43. https://doi.org/10.4236/ica.2017.81003

Received: December 1, 2016

Accepted: January 7, 2017

Published: January 10, 2017

Copyright (c) 2017 by authors and Scientific Research Publishing Inc. This work is licensed under the Creative Commons Attribution International License (CC BY 4.0).

http://creativecommons.org/licenses/by/4.0/

\begin{abstract}
This paper proposes the new speed control method of electric vehicles by generating the longitudinal speed pattern for improving the ride comfort against the longitudinal acceleration/deceleration. Since the longitudinal acceleration/ deceleration of EVs causes the discomfort of passengers, reducing such bad effect of acceleration/deceleration acting on passengers is very important for not only the ride comfort of passengers but also vehicle running safety. The proposed method is applying the general optimal control theory and it generates the speed pattern for improving the passenger ride quality based on evaluating the variations of acceleration and the jerk which is time derivative of the acceleration. The effectiveness of the present method is demonstrated through numerical experiments compared with some conventional methods.
\end{abstract}

\section{Keywords}

Electric Vehicle, Ride Quality, Speed Pattern, Acceleration/Deceleration, Jerk

\section{Introduction}

In recent years, with the wide spread of internal-combustion engine vehicles (ICEVs) all over the world, the environment and energy problems are going severely. Therefore, the development of next-generation vehicles such as hybrid vehicles (HVs) and electric vehicles (EVs) has been focused. Especially, EVs have attracted great interests as a powerful solution against the problems. EVs are automobiles which are propelled by electric motors, using electrical energy stored in batteries or another energy storage device. Electric motors have several advantages over ICEs [1] [2] [3] [4]:

- Energy efficient. Electric motors convert $75 \%$ of the chemical energy from the batteries to power the wheels-ICEs only convert $20 \%$ of the energy stored in 
gasoline.

- Environmentally friendly. EVs emit no tailpipe pollutants, although the power plant producing the electricity may emit them. Electricity from nuclear-, hydro-, solar-, wind-powered plants causes no air pollutants.

- The input/output response is faster than for gasoline/diesel engines. It is said that the motor torque response is 2 orders of magnitude faster than that of the engine. E.g., if engine torque response costs $500 \mathrm{~ms}$, the response time of motor toque will be $5 \mathrm{~ms}$.

- The torque generated in the wheels can be detected relatively accurately. For engine, the output torque varies along with the temperature and revolutions, even it has high-nonlinearity. Consequently, the value of torque is too difficult to be measured accurately. However, the value of motor torque is surveyed easily and accurately from the view of current control.

- The motor can be made small enough, then the vehicles can be made smaller by using multiple motors placed closer to the wheels. The drive wheels can be controlled fully and independently. E.g., it becomes easily achievable to control the differences of driving force developed between the left and right wheel.

From these good points of EVs, we can realize the superior running of vehicle with the good ride quality by using appropriate speed control. Such superior speed control is very useful for autonomous vehicle and some types of autonomous vehicle can also be applied, for example, PRT (Personal Rapid Transit) [5] and so on.

From the point of view of preventing a motion sickness and an accident, the ride comfort of the car is also very important [6]. Because vertical vibration influences the ride comfort of passengers, many studies are performed about the relation of vertical vibration and ride quality from the past few decades. For example, [7] or [8] are typical works for investigating the analyses, the ride discomfort caused by vertical vibration. Furthermore, an active suspension control system [9] is a typical control system for preventing the vertical vibration.

As well as the vertical vibration, it is important to reduce the influence on lateral speed change for improving overall ride quality. However, unfortunately, there are few studies on ride discomfort due to longitudinal acceleration/deceleration. Therefore, in this paper, we develop the speed control method by generating the longitudinal speed pattern using the jerk which is time derivative of the acceleration and the acceleration as the evaluation index, for improving the ride comfort against the longitudinal acceleration/deceleration. The method is applying the general optimal control theory and also based on the techniques proposed in [10] [11] [12] [13]. The method aims to contribute to improve the beginner driver's driving skill from the viewpoint of passenger's comfortability by showing the ideal running pattern and checking the driving.

The rest of this paper is organized as follows: Section 2 states the evaluation of ride comfort. In Section 3, the proposed generation method of speed pattern is presented. The extended proposed method is shown and simulation results are 
discussed in Section 4. Finally, we end the paper with some conclusions and future work in Section 5.

\section{Evaluation of Ride Quality}

There are various factors which have an influence on ride quality, but the index of the ride quality depends on individuals. About the railroad carriage, various research about the evaluation of the ride comfort with respect to the frequency of vibrations in vertical and horizontal directions have been reported in [14].

However, most of these deal with the evaluation of ride quality with respect to the sustained vibration of the steady run or vibration in the vertical direction. In the case of EVs, it is important to evaluate the ride comfort with respect to lateral speed change. In relation to this point, there are [15] and [16] as the study which investigated the relation between the acceleration/deceleration, the jerk (time derivative of the acceleration) and ride quality. In [15] and [16], subjectivity rating of the ride comfort tests about a start, a stop, immediate start, a run including the hitting the brakes for the resting posture and the reading posture and derive the following linear multiple regression model for the ride quality index.

$$
d(t)=\beta_{0}+\beta_{1} a_{p^{+}}(t)+\beta_{2} a_{p^{-}}(t)+\beta_{3} j_{r+}(t)+\beta_{4} j_{r-}(t)+\epsilon(t)
$$

where parameters in (1) are defined in Table 1. And where $\boldsymbol{a}_{p^{+}}(\boldsymbol{t}), \boldsymbol{a}_{p^{-}}(\boldsymbol{t})$, $\boldsymbol{j}_{\boldsymbol{r}+}(\boldsymbol{t}), \quad \boldsymbol{j}_{\boldsymbol{r}-}(\boldsymbol{t})$ in $T=(t-3, t)$ are given as follows.

$$
\begin{gathered}
\boldsymbol{a}_{\boldsymbol{p}^{+}}(\boldsymbol{t})= \begin{cases}\max _{t \in T} a(t), & \left|\max _{t \in T} a(t)\right| \geq\left|\min _{t \in T} a(t)\right| \\
0, & \left|\max _{t \in T} a(t)\right|<\left|\min _{t \in T} a(t)\right|\end{cases} \\
\boldsymbol{a}_{p^{-}}(\boldsymbol{t})= \begin{cases}0, & \left|\max _{t \in T} a(t)\right| \geq\left|\min _{t \in T} a(t)\right| \\
\min _{t \in T} a(t), & \left|\max _{t \in T} a(t)\right|<\left|\min _{t \in T} a(t)\right|\end{cases} \\
\boldsymbol{j}_{\boldsymbol{r}^{+}}(\boldsymbol{t})= \begin{cases}\sqrt{\frac{1}{3} \int_{t-3}^{t} j^{2}(\tau) \mathrm{d} \tau,}, \boldsymbol{j}(\boldsymbol{T}) \geq 0 \\
0, & \boldsymbol{j}(\boldsymbol{T})<0\end{cases}
\end{gathered}
$$

\begin{tabular}{|c|c|}
\hline$d(t)$ & ride comfort index at time $t$ \\
\hline$a_{p+}(t)$ & peak value of acceleration in $T=(t-3, t)$ ( 3 seconds just before time $t)$ \\
\hline$a_{p-}(t)$ & peak absolute value of deceleration in $T$ \\
\hline$j(T)$ & average value of jerk in $T$ \\
\hline$j_{r+}(t)$ & effective value of jerk in the case of positive value of $j(T)$ \\
\hline$j_{r-}(t)$ & effective value of jerk in the case of negative value of $j(T)$ \\
\hline$\epsilon(t)$ & error term \\
\hline$\beta_{0}$ & constant term \\
\hline$\beta_{k}$ & partial regression coefficient \\
\hline
\end{tabular}

Table 1. Definition of parameters. 


$$
\boldsymbol{j}_{r-}(\boldsymbol{t})= \begin{cases}0, & \boldsymbol{j}(\boldsymbol{T}) \geq 0 \\ \sqrt{\frac{1}{3} \int_{t-3}^{t} j^{2}(\tau) \mathrm{d} \tau}, & \boldsymbol{j}(\boldsymbol{T})<0\end{cases}
$$

Unfortunately, it is not possible to derive the speed pattern by using $d(t)$ directly since the $d(t)$ is ride comfort index at the specific time $t$ derived based on the acceleration and the jerk in the real time. It can't show overall evaluation. Then we need to consider another index for speed control for overall ride comfort. In [15] and [16], we can see that the value of deceleration, the value of jerk in deceleration, the value of jerk in acceleration and the value of acceleration have big influence on the ride quality by their order.

Therefore, it is important to suppress both of the acceleration/deceleration and the jerk to improve the ride quality and we construct the speed control method on these facts.

\section{Speed Control Method}

\subsection{Generation of Speed Pattern}

It's important for speed control method to consider the speed pattern. The speed pattern is defined as the ideal speed plan to satisfy various demands/limits for ride comfort, energy efficiency at acceleration, position, time and so on. In [17], the speed pattern is derived based on the SMART control method [18] by using following evaluation function.

$$
J_{0}=\int_{0}^{t_{f}}\left(\frac{\mathrm{d} a}{\mathrm{~d} t}\right)^{2} \mathrm{~d} t
$$

where $a$ is the acceleration and $t_{f}$ is the terminal time, and where the statespace vehicle model is as

$$
\left(\begin{array}{c}
\dot{v} \\
\dot{a}
\end{array}\right)=\left(\begin{array}{ll}
0 & 1 \\
0 & 0
\end{array}\right)\left(\begin{array}{l}
v \\
a
\end{array}\right)+\left(\begin{array}{l}
0 \\
1
\end{array}\right) u
$$

However, the acceleration, which is one of the most important factor influenced to the ride quality as I mentioned in Section 2, does not include directly in this evaluation function. In addition, a vehicle position is not included in this model. It's important factor for realizing the automatic driving in the near future. From these facts, the state space model and the evaluation function in this paper is defined as follows.

$$
\begin{aligned}
\left(\begin{array}{c}
\dot{x} \\
\dot{v} \\
\dot{a}
\end{array}\right) & =\left(\begin{array}{lll}
0 & 1 & 0 \\
0 & 0 & 1 \\
0 & 0 & 0
\end{array}\right)\left(\begin{array}{l}
x \\
v \\
a
\end{array}\right)+\left(\begin{array}{l}
0 \\
0 \\
1
\end{array}\right) u \\
J_{1} & =\int_{0}^{t_{f}}\left[\left(\frac{\mathrm{d} a}{\mathrm{~d} t}\right)^{2}+(q a)^{2}\right] \mathrm{d} t
\end{aligned}
$$

where (8) is written as $\dot{\boldsymbol{x}}=\boldsymbol{A} x+\boldsymbol{b} u$ with;

$$
\boldsymbol{x}=\left(\begin{array}{l}
x \\
v \\
a
\end{array}\right), \boldsymbol{A}=\left(\begin{array}{lll}
0 & 1 & 0 \\
0 & 0 & 1 \\
0 & 0 & 0
\end{array}\right) \text { and } \boldsymbol{B}=\left(\begin{array}{l}
0 \\
0 \\
1
\end{array}\right) \text {. }
$$


It's also possible to control the vehicle position to add $\boldsymbol{x}$ in the state space variables for automatic driving. Furthermore, we can derive the speed pattern which emphasized the ride comfort against the acceleration and deceleration by adding the weighted $a$ to evaluation function. ( $q$ is the weighting constant.)

Then, by using the generalized optimal control theory, we can derive the following Hamiltonian $H$ from (8) and (9).

$$
H=\frac{1}{2}\left(u^{2}+a^{2}\right)+\lambda^{\mathrm{T}}(A x+B u)=\frac{1}{2}\left(u^{2}+x^{\mathrm{T}} Q x\right)+\lambda^{\mathrm{T}}(A x+B u)
$$

where $\lambda$ is the Lagrange multiplier and where

$$
Q=\left(\begin{array}{ccc}
0 & 0 & 0 \\
0 & 0 & 0 \\
0 & 0 & q^{2}
\end{array}\right)
$$

The solution minimized $J_{1}$ is obtained from $\frac{\partial H}{\partial u}=0$ as

$$
u=-b^{\mathrm{T}} \lambda
$$

From $\dot{x}=\frac{\partial H}{\partial \lambda}$ and $\dot{\lambda}=-\frac{\partial H}{\partial x}$, we can obtain

$$
\left(\begin{array}{l}
\dot{x} \\
\dot{\lambda}
\end{array}\right)=\left(\begin{array}{cc}
A & -B B^{\mathrm{T}} \\
-Q & -A^{\mathrm{T}}
\end{array}\right)\left(\begin{array}{l}
x \\
\lambda
\end{array}\right)
$$

This equation is expressed as

$$
\left(\begin{array}{c}
\dot{x} \\
\dot{v} \\
\dot{a} \\
\dot{\lambda}_{1} \\
\dot{\lambda}_{2} \\
\dot{\lambda}_{3}
\end{array}\right)=\left(\begin{array}{cccccc}
0 & 1 & 0 & 0 & 0 & 0 \\
0 & 0 & 1 & 0 & 0 & 0 \\
0 & 0 & 0 & 0 & 0 & -1 \\
0 & 0 & 0 & 0 & 0 & 0 \\
0 & 0 & 0 & -1 & 0 & 0 \\
0 & 0 & -q^{2} & 0 & -1 & 0
\end{array}\right)\left(\begin{array}{c}
x \\
v \\
a \\
\lambda_{1} \\
\lambda_{2} \\
\lambda_{3}
\end{array}\right)
$$

From (14),

$$
\dot{\lambda}_{2}=-C^{\prime \prime}
$$

is obtained. Where $C^{\prime \prime}$ is given constant. Furthermore, the following state space equation is derived.

$$
\left(\begin{array}{l}
\dot{a} \\
\dot{\lambda}_{2} \\
\dot{\lambda}_{3}
\end{array}\right)=\left(\begin{array}{ccc}
0 & 0 & -1 \\
0 & 0 & 0 \\
-q^{2} & -1 & 0
\end{array}\right)\left(\begin{array}{c}
a \\
\lambda_{2} \\
\lambda_{3}
\end{array}\right)+\left(\begin{array}{c}
0 \\
-C^{\prime \prime} \\
0
\end{array}\right)
$$

The time response of this system expressed as (16) is

$$
\boldsymbol{x}_{1}(t)=\mathrm{e}^{A_{1} t} \boldsymbol{x}_{1}(0)+\int_{0}^{t} \mathrm{e}^{A_{1}(t-\tau)} B_{1} u_{1}(\tau) \mathrm{d} \tau
$$

where

$$
\boldsymbol{x}_{1}=\left(\begin{array}{c}
\dot{a} \\
\dot{\lambda}_{2} \\
\dot{\lambda}_{3}
\end{array}\right), \boldsymbol{A}_{1}=\left(\begin{array}{ccc}
0 & 0 & -1 \\
0 & 0 & 0 \\
-q^{2} & -1 & 0
\end{array}\right), \boldsymbol{B}_{1}=\left(\begin{array}{c}
0 \\
-C^{\prime \prime} \\
0
\end{array}\right) \text { and } \boldsymbol{u}_{1}=1
$$


Then, from (17), we can derive $\boldsymbol{x}_{1}(t)$ as follows.

$$
\begin{aligned}
\left(\begin{array}{l}
a \\
\lambda_{2} \\
\lambda_{3}
\end{array}\right)= & \frac{1}{2}\left(\begin{array}{ccc}
\left(\mathrm{e}^{q t}+\mathrm{e}^{-q t}\right) & \frac{1}{q^{2}}\left(-2+\mathrm{e}^{q t}+\mathrm{e}^{-q t}\right) & \frac{1}{q}\left(-\mathrm{e}^{q t}+\mathrm{e}^{-q t}\right) \\
0 & 1 & 0 \\
q\left(-\mathrm{e}^{q t}+\mathrm{e}^{-q t}\right) & \frac{1}{q}\left(-\mathrm{e}^{q t}+\mathrm{e}^{-q t}\right) & \left(\mathrm{e}^{q t}+\mathrm{e}^{-q t}\right)
\end{array}\right) \\
& \times\left(\begin{array}{l}
a(0) \\
\lambda_{2}(0) \\
\lambda_{3}(0)
\end{array}\right)+\frac{-C^{\prime \prime}}{2}\left(\begin{array}{c}
\frac{1}{q^{2}}\left(-2 t+\frac{1}{q} \mathrm{e}^{q t}-\frac{1}{q} \mathrm{e}^{-q t}\right) \\
2 t \\
\frac{1}{q}\left(\frac{2}{q}-\frac{1}{q} \mathrm{e}^{q t}-\frac{1}{q} \mathrm{e}^{q t}\right)
\end{array}\right)
\end{aligned}
$$

Also $a(t)$ is obtained as follows.

$$
\begin{aligned}
a(t)= & \left(\frac{1}{2} a(0)+\frac{1}{2 q^{2}} \lambda_{2}(0)-\frac{1}{2 q} \lambda_{3}(0)-\frac{C^{\prime \prime}}{2 q^{3}}\right) \mathrm{e}^{q t} \\
& +\left(\frac{1}{2} a(0)+\frac{1}{2 q^{2}} \lambda_{2}(0)+\frac{1}{2 q} \lambda_{3}(0)+\frac{C^{\prime \prime}}{2 q^{3}}\right) \mathrm{e}^{-q t}+\frac{C^{\prime \prime}}{q^{2}} t+\frac{\lambda_{2}(0)}{q^{2}}
\end{aligned}
$$

where $q, a(0), \lambda_{2}(0), \lambda_{3}(0), C^{\prime \prime}$ are given constants. Expressing constant terms in (19) as $C_{j}^{\prime \prime}(j=0,1,2,3),(19)$ is described as follows.

$$
a(t)=C_{0}^{\prime \prime} \mathrm{e}^{q t}+C_{1}^{\prime \prime} \mathrm{e}^{-q t}+C_{2}^{\prime \prime} t+C_{3}^{\prime \prime}
$$

Finally we can derive the following speed pattern after deformation of equations with state space equation: $\dot{x}=\frac{\partial H}{\partial \lambda}$, co-state space equation: $\dot{\lambda}=-\frac{\partial H}{\partial x}$ and stationarity equation: $0=-\frac{\partial H}{\partial u}$.

$$
\begin{aligned}
& x(t)=C_{0} \mathrm{e}^{q t}+C_{1} \mathrm{e}^{-q t}+C_{2} t^{3}+C_{3} t^{2}+C_{4} t+C_{5} \\
& v(t)=q C_{0} \mathrm{e}^{q t}-q C_{1} \mathrm{e}^{-q t}+3 C_{2} t^{2}+2 C_{3} t+C_{4} t \\
& a(t)=q^{2} C_{0} \mathrm{e}^{q t}+q^{2} C_{1} \mathrm{e}^{-q t}+6 C_{2} t+2 C_{3}
\end{aligned}
$$

where $C_{j}(j=0,1,2,3,4,5)$ are constant coefficients. These values can be decided from initial and terminal conditions of $t, x, v$ and $a$.

This speed pattern includes the method by [17]. This fact is shown as follows. Firstly, since the evaluation function of the proposed method, (9), is added $(q a)^{2}$ to the one using in [17], (6), we can see that both equations are same if $q$ $=0$. But, the speed pattern is unable to derived (it should be indefinite solution) if $q=0$. Then, let's do Maclaurin's expansion of the proposed speed pattern with neighborhood of $q=0$ and derive the 4-th order approximated polynomial, which same order of [17].

One example is shown. Let's consider the following initial and terminal conditions.

$$
\left\{\begin{array}{l}
t_{0}=0: x_{0}=0, v_{0}=10, a_{0}=1 \\
t_{f}=10: x_{f}=100, v_{f}=0, a_{f}=0
\end{array}\right.
$$

These conditions show the situation that the vehicle runs from a state running 
in speed $10 \mathrm{~m} / \mathrm{s}$ and acceleration $1 \mathrm{~m} / \mathrm{s}^{2}$ to the $100 \mathrm{~m}$ spot ten seconds later, and to stop.

Figure 1 shows the difference values of between the proposed speed pattern and the one in [17] is $-3 \times 10^{-14} \sim 8 \times 10^{-14}$. It may be considered to be the same, not little difference. Therefore, we can see that the proposed method includes and extends the method in [17].

In Figure 2 and Figure 3, red line indicates the result by the conventional method in [17] and blue line indicates the result by the proposed method with $q$ $=3.5$ which minimized total sum of $d(t)$. Figure 2 shows the time response of vehicle speed and acceleration respectively. From this figure, we can see that

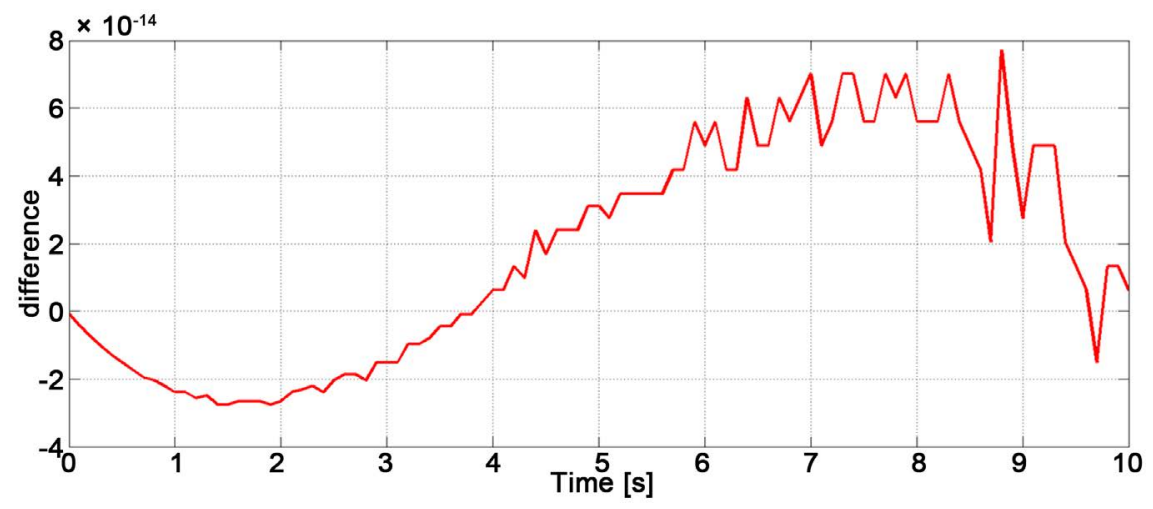

Figure 1. Difference values of speed pattern between the proposed one and [17].

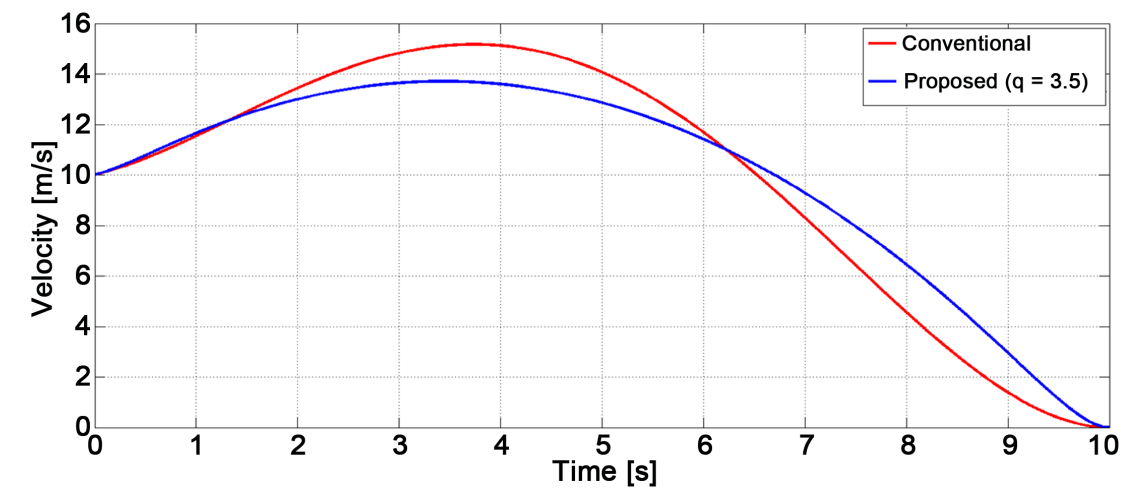

(a)

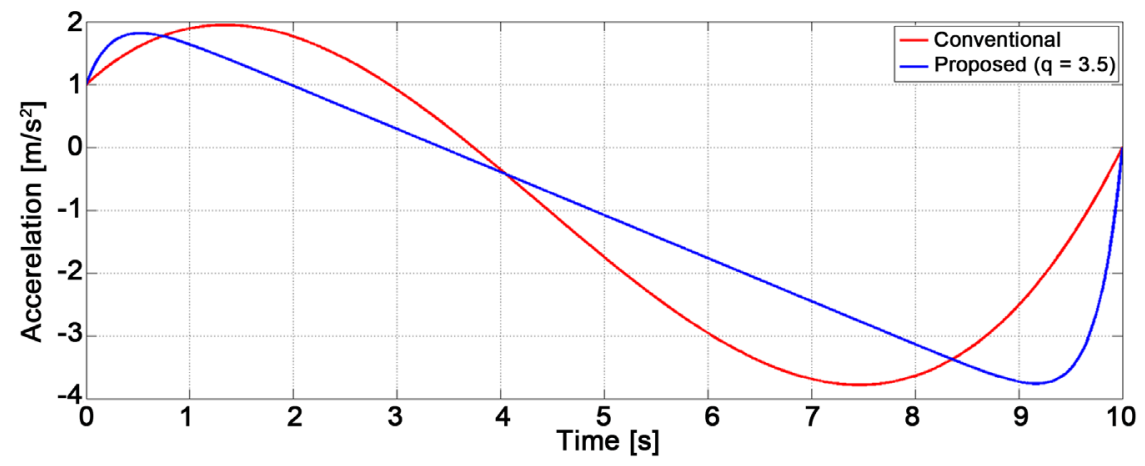

(b)

Figure 2. Time response of (a) vehicle speed and (b) acceleration. 


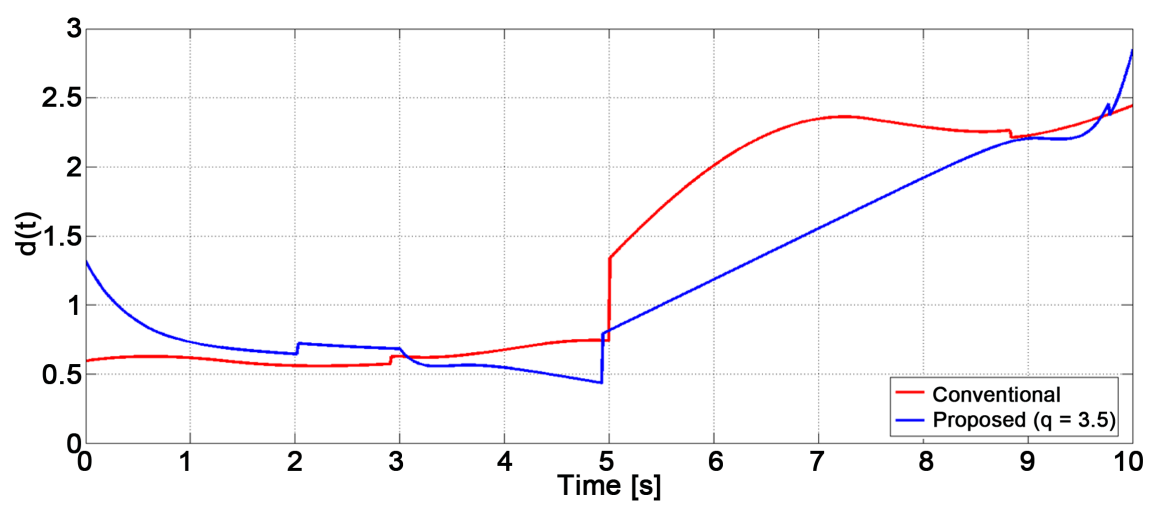

Figure 3. Time response of ride comfort index $d(t)$.

both methods (the proposed and the method in [17] can generate the speed pattern satisfied initial and terminal conditions. Figure 3 shows time response of $d(t)$. We can see that the proposed method can suppress the value of $d(t)$ lower than the method in [17] in the most part of the whole running period. Therefore, we find out that the proposed method can improve the ride comfort than the method in [17].

\subsection{Extended Generation Method}

The proposed method is extended to the flexible generation method which can cope with the change of terminal conditions in the way of the run for practical use. For example, the method is extended to be able to deal with the situation that it is necessary to shorten a stop spot by some kind of factors such as other vehicles getting into the way. In such situation, the speed pattern should be re-generated flexibly in real-time accordance with the change of conditions.

As the result of many simulations, we see that the remaining run time after the pattern re-generated greatly influenced the quality of ride comfort. Since the change of the run time brings the sudden change of the jerk. Therefore, the following evaluation function $\left(J_{2}\right)$ is introduced to decide appropriate remaining run time.

$$
J_{2}=r x+s y
$$

where $x$ is the absolute value of the difference of the value of the jerk just before and after the pattern re-generated, $y$ is the absolute value of the difference of the derivative value of the jerk just before and after the pattern change, and $r, s$ are constant weights. Because it was a problem that the jerk suddenly changes in before and after the pattern re-generated, appropriate values of the weight $q$ of $J_{1}$ in (9) and the remaining run time are decided by using this evaluation function $J_{2}$ in (23) for the change of jerk consecutively and smoothly as much as possible. A flow of rough processing of this method is shown in Figure 4.

In addition, a search range at remaining run time is set. For example, the search range is set as not exceeding the whole running time set beforehand. If the running distance becomes long, remaining run time is able to be increased and search the best values of $q$ and the remaining run time in the enlarged range. 


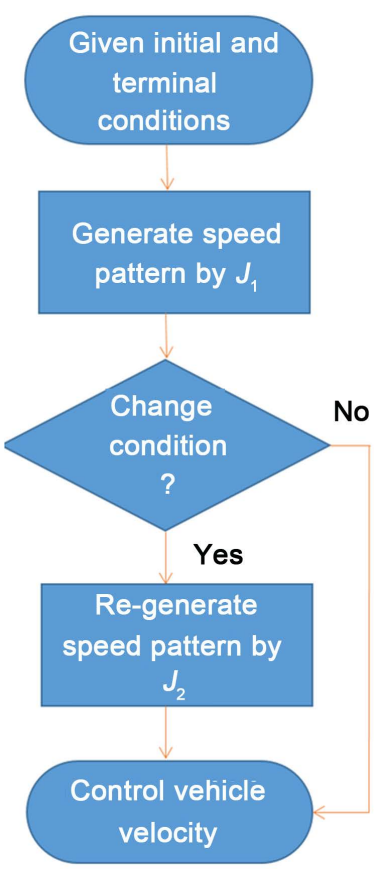

Figure 4. Flow of rough processing of the proposed method.

Then, it becomes possible to derive the speed pattern with best ride comfort, which minimize the total $d(t)$ in whole running time, against the change of terminal conditions.

\section{Numerical Examples}

Let's confirm the effectiveness of the proposed extended method in some simulations.

\subsection{Simulation I}

Firstly, let's consider the situation that the stop spot is shortened after starting off. The first condition is as follows.

$$
\left\{\begin{array}{l}
t_{0}=0: x_{0}=0, v_{0}=0, a_{0}=0 \\
t_{f}=10: x_{f}=100, v_{f}=0, a_{f}=0
\end{array}\right.
$$

Then, at running $60 \mathrm{~m}$ spot, the stop spot is shortened from $100 \mathrm{~m}$ to $70 \mathrm{~m}$ as follows.

$$
\left\{\begin{array}{l}
t_{0}=0: x_{0}=60, v_{0}=v_{s}, a_{0}=a_{s} \\
t_{f}=[\text { search }]: x_{f}=70, v_{f}=0, a_{f}=0
\end{array}\right.
$$

where $v_{s}$ is final speed value before re-generate the speed pattern and $a_{s}$ is final acceleration value before re-generate the pattern.

The results by the proposed method are shown as Figure 5 and Figure 6. In this simulation, the pattern is re-generated at $5.61 \mathrm{~s}$, weights are $q=2.2, r=1, s$ $=0$ and remaining run time after regeneration of pattern is $1.00 \mathrm{~s}$. So, we can see that the method can cope with the sudden change of stop spot from Figure 5. From Figure 6, the ride comfort becomes worse at the time of shortening the 


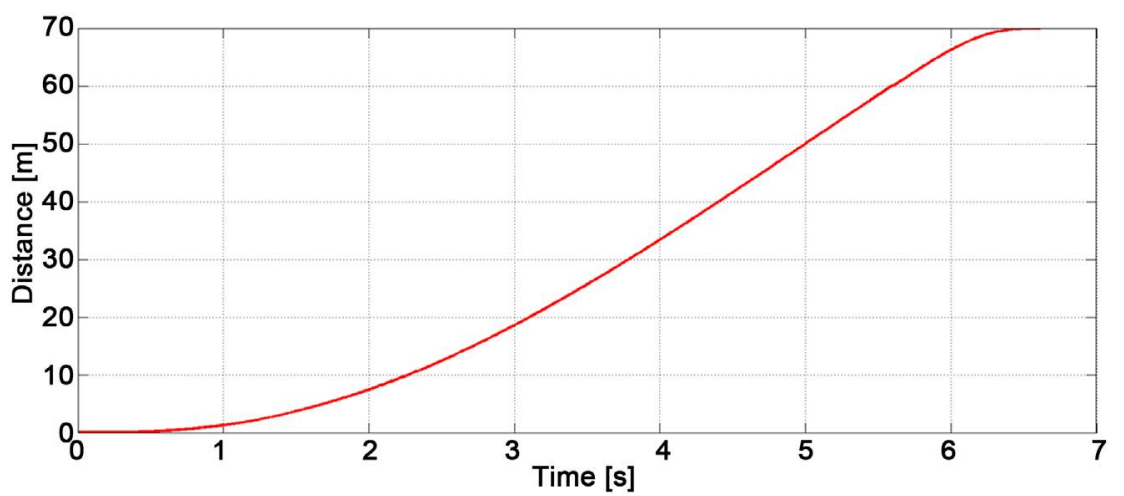

(a)

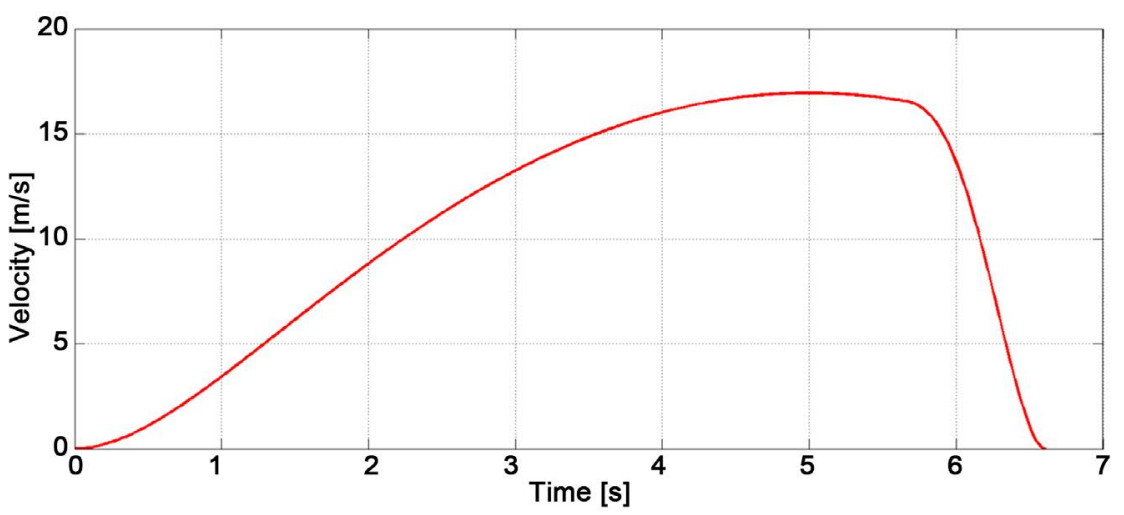

(b)

Figure 5. Time response of (a) vehicle position and (b) speed acceleration (Simulation I).

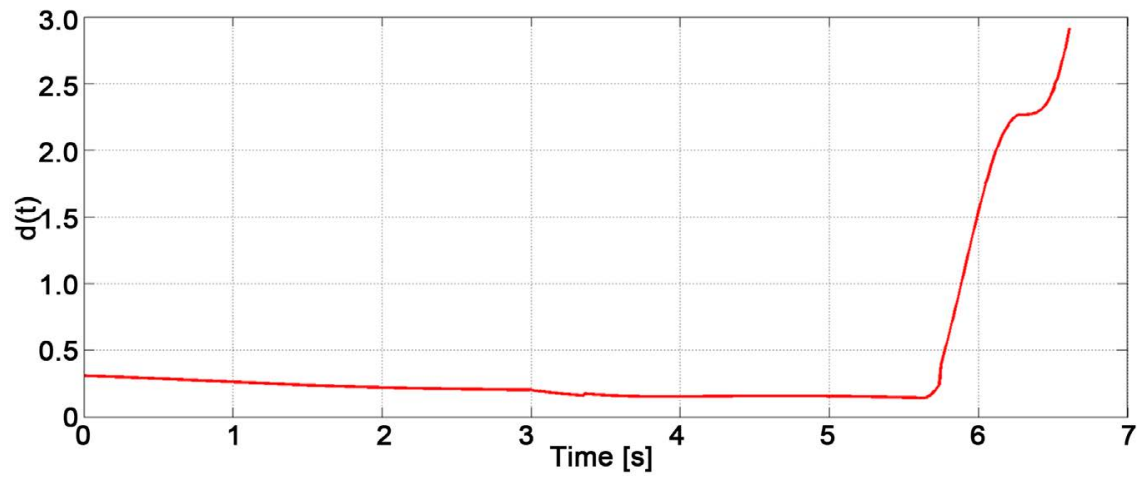

Figure 6. Time response of ride comfort index $d(t)$ (Simulation I).

stop spot at $5.61 \mathrm{~s}$. But it's natural response due to the sudden shortening of stop distance for safety. Therefore, we can say that the proposed method shows the good performance totally.

\subsection{Simulation II}

Let's consider the situation that the stop spot is lengthened after starting off.

The first conditions as follows.

$$
\left\{\begin{array}{l}
t_{0}=0: x_{0}=0, v_{0}=0, a_{0}=0 \\
t_{f}=10: x_{f}=100, v_{f}=0, a_{f}=0
\end{array}\right.
$$


Then, at running $60 \mathrm{~m}$ spot, the stop spot is lengthened from $100 \mathrm{~m}$ to $130 \mathrm{~m}$ as follows.

$$
\left\{\begin{array}{l}
t_{0}=0: x_{0}=60, v_{0}=v_{s}, a_{0}=a_{s} \\
t_{f}=[\text { search }]: x_{f}=130, v_{f}=0, a_{f}=0
\end{array}\right.
$$

The results by the proposed method are shown in Figure 7. In this simulation, the pattern is re-generated at $5.61 \mathrm{~s}$, weights are $q=1.9, r=1, s=0$ and remaining run time after pattern re-generated is $8.173 \mathrm{~s}$. From this figure, we can see that the method can cope with the situation of lengthening the stop spot.

From Figure 8, the ride comfort index $d(t)$ turns worse suddenly at about 6.9 s. This is because the absolute value of the acceleration is bigger here, and it has a big influence on the $d(t)$. But, after $6.9 \mathrm{~s}$ the value of $d(t)$ is suppressed gradually. Therefore, the proposed method shows the good performance.

\subsection{Simulation III}

In this simulation, let's consider the situation that the stop spot is changed two times.

The first conditions as follows.

$$
\left\{\begin{array}{l}
t_{0}=0: x_{0}=0, v_{0}=0, a_{0}=0 \\
t_{f}=10: x_{f}=100, v_{f}=0, a_{f}=0
\end{array}\right.
$$

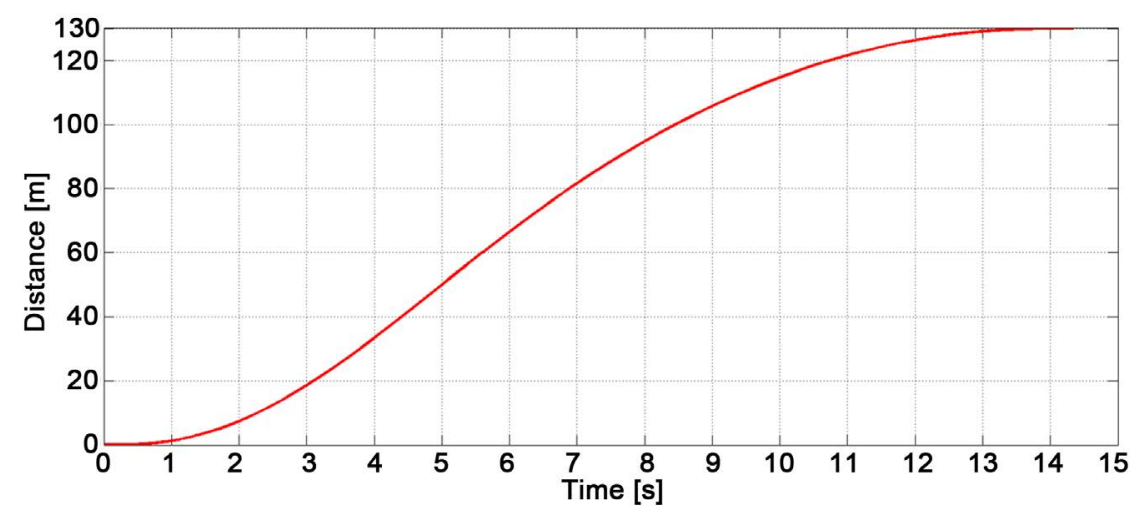

(a)

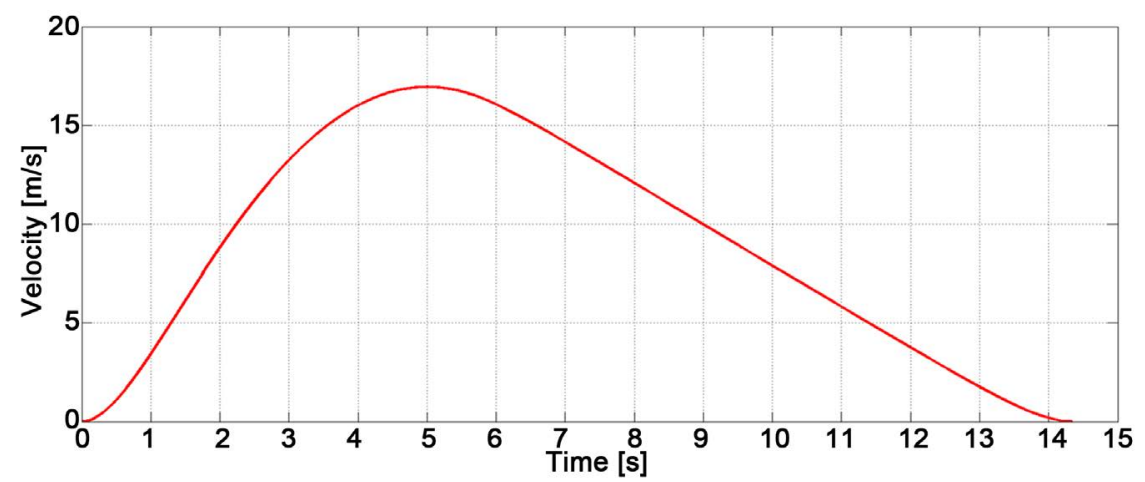

(b)

Figure 7. Time response of (a) vehicle position and (b) speed acceleration (Simulation II). 


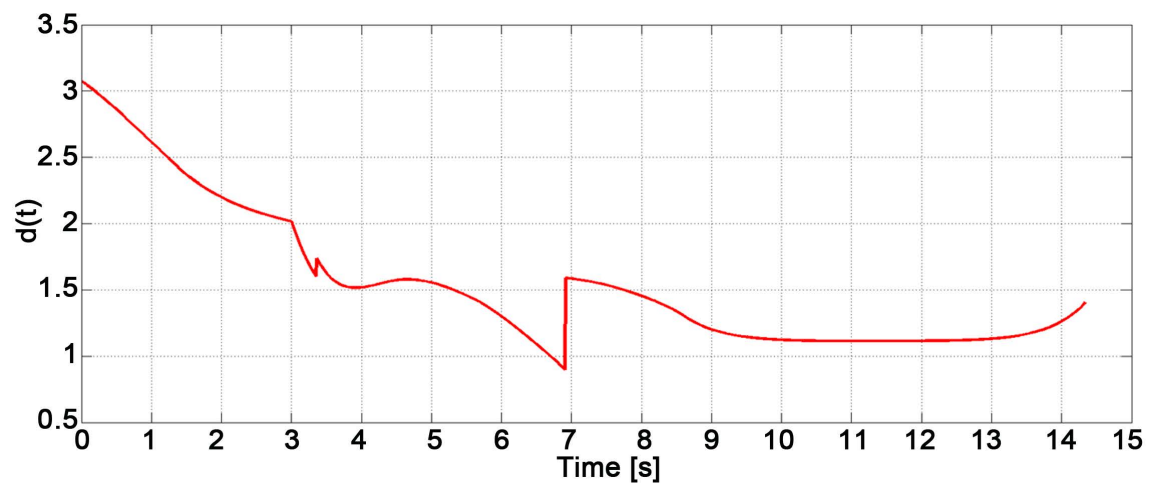

Figure 8. Time response of ride comfort index $d(t)$ (Simulation II).

Firstly, at running $60 \mathrm{~m}$ spot, the stop spot is lengthened from $100 \mathrm{~m}$ to $130 \mathrm{~m}$ as follows.

$$
\left\{\begin{array}{l}
t_{0}=0: x_{0}=60, v_{0}=v_{s}, a_{0}=a_{s} \\
t_{f}=[\text { search }]: x_{f}=130, v_{f}=0, a_{f}=0
\end{array}\right.
$$

Then, at running $100 \mathrm{~m}$ spot, the stop spot is shortened from $130 \mathrm{~m}$ to $120 \mathrm{~m}$ as follows.

$$
\left\{\begin{array}{l}
t_{0}=0: x_{0}=100, v_{0}=v_{s}, a_{0}=a_{s} \\
t_{f}=[\operatorname{search}]: x_{f}=120, v_{f}=0, a_{f}=0
\end{array}\right.
$$

The results by the proposed extended method are shown in Figure 9. In this simulation, the pattern is re-generated at $5.61 \mathrm{~s}$ and $8.36 \mathrm{~s}$. The weights are $q=$ 1.9, $r=1, s=0$ and $q=4.1, r=0.4, s=0.2$ respectively. Total running time is $12.11 \mathrm{~s}$.

From this figure, we can see that the method can cope with the situation of change the stop spot two time.

From Figure 10, the ride comfort index $d(t)$ turns worse slightly at about 7.0 $\mathrm{s}$. This is because the absolute value of the acceleration is bigger here, and it has a big influence on the $d(t)$. Since the condition is changed again at $8.3 \mathrm{~s}, d(t)$ turns worse gradually after this time due to the influence of suddenly shortened stop point.

\subsection{Simulation IV}

In this section, the proposed extended method is compared with the conventional method [17] in the situation that the stop spot is shortened after starting off.

$$
\left\{\begin{array}{l}
t_{0}=0: x_{0}=0, v_{0}=0, a_{0}=0 \\
t_{f}=10: x_{f}=100, v_{f}=0, a_{f}=0
\end{array}\right.
$$

Then, at running $40 \mathrm{~m}$ spot, the stop spot is shortened from $100 \mathrm{~m}$ to $90 \mathrm{~m}$ as follows.

$$
\left\{\begin{array}{l}
t_{0}=0: x_{0}=40, v_{0}=v_{s}, a_{0}=a_{s} \\
t_{f}=[\text { search }]: x_{f}=90, v_{f}=0, a_{f}=0
\end{array}\right.
$$




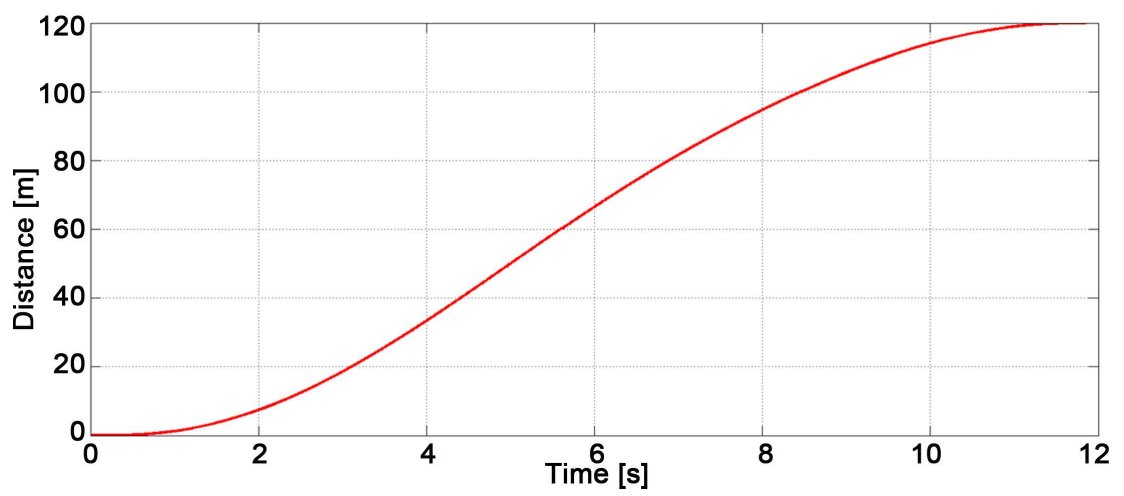

(a)

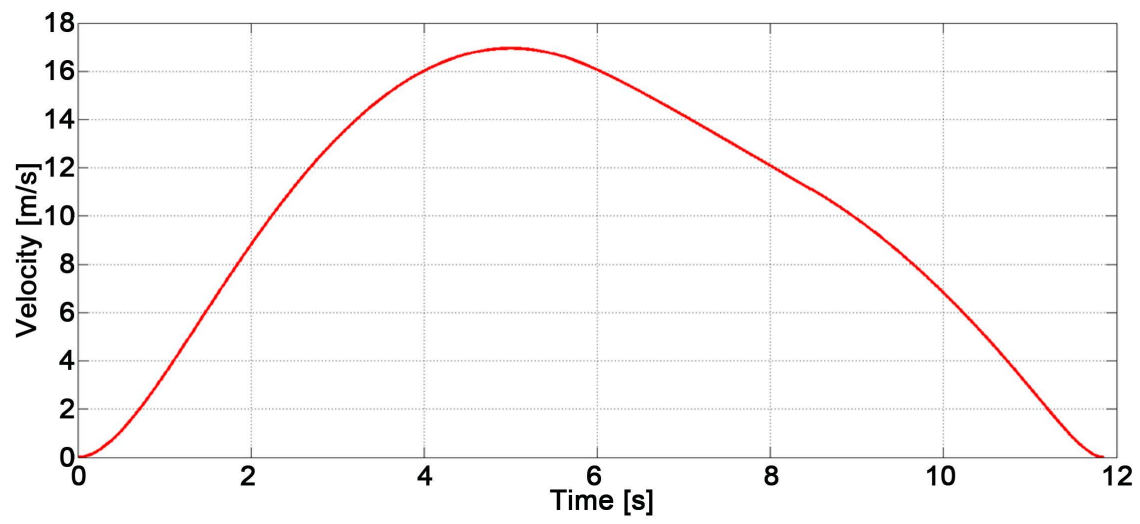

(b)

Figure 9. Time response of (a) vehicle position and (b) speed acceleration (Simulation III).

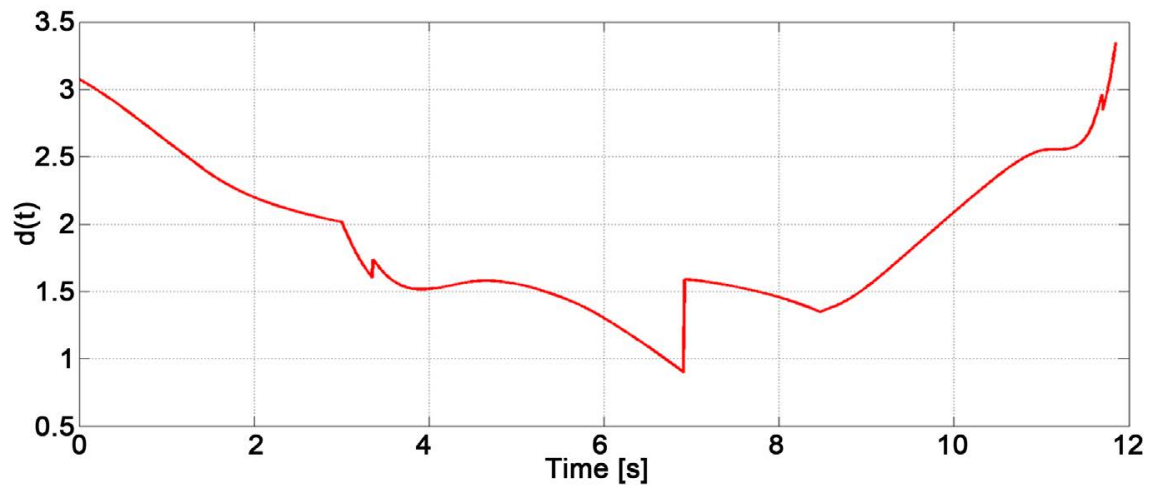

Figure 10. Time response of ride comfort index $d(t)$ (Simulation III).

The result of comfort index $d(t)$ is shown as Figure 11. In this simulation, the conventional method got worse the ride quality after situation changes. On the other hand, the proposed extended method can cope with the change. So, we can see the good performance of the proposed method.

\section{Conclusions}

In this paper, we have proposed the speed control method based on general optimal control theory for improving the passenger ride comfort of electric vehicles. 


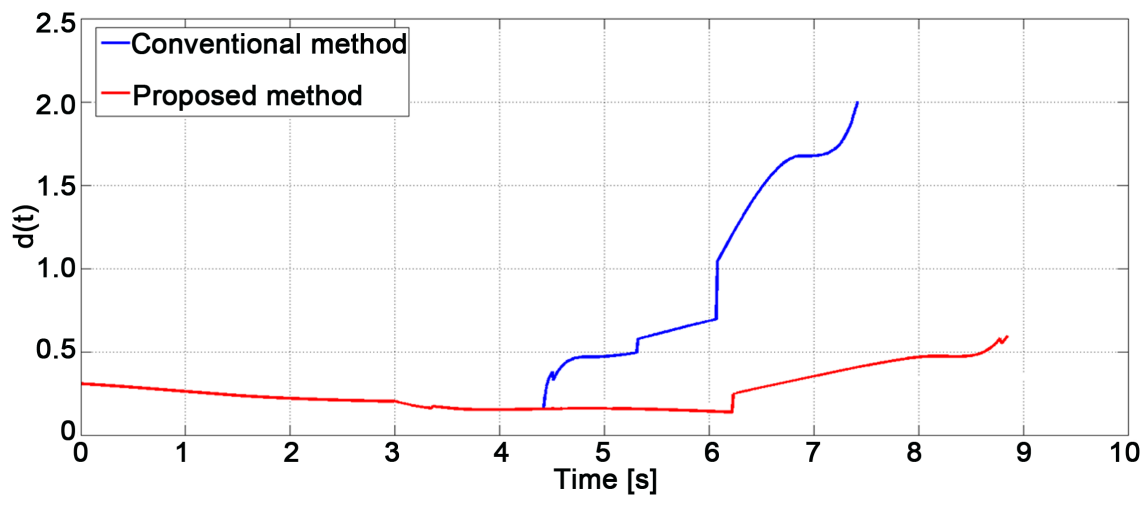

Figure 11. Time response of ride comfort index $d(t)$ (Simulation IV).

The method is applying the general optimal control theory and also based on the conventional techniques. Furthermore, it includes the conventional method and extends the flexible speed-pattern-generation method which can cope with the change of terminal conditions in the way of the run. The method also aims to contribute to improving the beginner driver's driving skill from the viewpoint of passenger's comfortability by showing the ideal running pattern and checking the driving. The proposed method can expect to be also useful for the run which emphasized ride comfort of the automatic operation car which would come to practical use in the future. It can also be applied to some types of autonomous vehicle, transit system, for example, PRT, and so on.

In future work, the suitability of the method must be studied not only the longitudinal run but also for overall driving situations. Furthermore, it is necessary to verify the effectiveness by actual experiments. Let's confirm the effectiveness.

\section{References}

[1] Brown, S., Pyke, D. and Steenhof, P. (2010) Electric Vehicles: The Role and Importance of Standards in an Emerging Market. Energy Policy, 38, 3797-3806. https://doi.org/10.1016/j.enpol.2010.02.059

[2] Mousazadeh, H., Keyhani, A., Mobli, H., Bardi, U., Lombardi, G. and Asmar, T. (2009) Environmental Assessment of Ramses Multipurpose Electric Vehicle Compared to a Conventional Combustion Engine Vehicle. Journal of Cleaner Production, 17, 781-790. https://doi.org/10.1016/j.jclepro.2009.01.004

[3] Hirota, T., Ueda, M. and Futami, T. (2011) Activities of Electric Vehicles and Prospect for Future Mobility. Journal of the Society of Instrument and Control Engineering, 50, 165-170.

[4] Tseng, H., Wu, J. and Liu, X. (2013) Affordability of Electric Vehicle for a Sustainable Transport System: An Economic and Environmental Analysis. Energy Policy, 61, 441-447. https://doi.org/10.1016/j.enpol.2013.06.026

[5] Janic, M. (2014) Advanced Transport Systems-Analysis, Modeling, and Evaluation of Performances. Springer-Verlag, London.

[6] Nozaki, H. (2008) Basic Automobile Engineering. Tokyo Denki University Press, Tokyo. (In Japanese)

[7] Akatsu, Y. (1998) An Evaluation Method of Improving Ride Comfort. The Journal of Society of Automotive Engineers of Japan, 67, 29-34. (In Japanese) 
[8] Cucuz, S. (1994) Evaluation of Ride Comfort. International Journal of Vehicle Design, 52, 318-325.

[9] Itagaki, N., Kinoshita, T. and Fukao, T. (2013) Semi-Active Suspension Control for Suppressing the Unsprung Mass Vibration and Improving the Ride Comfort. The Journal of Society of Automotive Engineers of Japan, 67, 29-34. (In Japanese)

[10] Guarino, C., Bianco, L., Piazzi, A. and Romano, R. (2004) Velocity Planning for Autonomous Vehicles. Proceedings of IEEE Intelligent Vehicles Symposium 2014, Parma, 14-17 June 2016, 413-418.

[11] Solea, R. and Nunes, U. (2006) Trajectory Planning with Velocity Planner for Fully-Automated Passenger Vehicles. Proceedings of IEEE Intelligent Transportation Systems Conference, Toronto, 17-20 September 2006, 474-480.

https://doi.org/10.1109/ITSC.2006.1706786

[12] Villagra, J., Milan'es, V., P'erez, J. and Godoy, J. (2012) Smooth Path and Speed planning for an Automated Public Transport Vehicle. Robotics and Autonomous Systems, 60, 252-265. https://doi.org/10.1016/j.robot.2011.11.001

[13] Lini, G., Piazzi, A. and Consolini, L. (2013) Affordability of Electric Vehicle for a Sustainable Transport System: An Economic and Environmental Analysis. International Journal of Control, Automation, and Systems, 11, 805-814. https://doi.org/10.1007/s12555-011-0065-y

[14] Nakagawa, C. (2010) Think about the Ride Comfort of the Rapid Transit Railway. Railway Research Review, 5, 19-21. (In Japanese)

[15] Wang, F., Sagawa, K. and Inooka, N. (2000) A Study of the Relationship between the Longitudinal Acceleration/Deceleration of Automobiles and Ride Comfort. Ergonomics (Japan Ergonomics Society Journal), 36, 191-200. (In Japanese) https://doi.org/10.5100/jie.36.191

[16] Wang, F., Sagawa, K., Ishihara, T. and Inooka, N. (2002) An Automobile Driver Assistance System for Improving Passenger Ride Comfort. The Transactions of the Institute of Electrical Engineers of Japan D, 122, 730-735. (In Japanese)

[17] Zhao, L. and Hori, Y. (2006) Real Time Smart Speed Pattern Generator for EVs to Improve Safety and Ride Comfort Taking Driver's Command Change and Limits of Acceleration and Jerk into Account. IEEJ Record of Industrial Measurement and Control, IIC-06-017.

[18] Mizoshita, Y., Hasegawa, S. and Takaishi, K. (1996) Vibration Minimized Access Control for Disk Drives. IEEE Transaction on Magnetics, 32, 1793-1798.

https://doi.org/10.1109/20.492867 
Submit or recommend next manuscript to SCIRP and we will provide best service for you:

Accepting pre-submission inquiries through Email, Facebook, LinkedIn, Twitter, etc. A wide selection of journals (inclusive of 9 subjects, more than 200 journals)

Providing 24-hour high-quality service

User-friendly online submission system

Fair and swift peer-review system

Efficient typesetting and proofreading procedure

Display of the result of downloads and visits, as well as the number of cited articles Maximum dissemination of your research work

Submit your manuscript at: http://papersubmission.scirp.org/

Or contact ica@scirp.org 